\title{
Pyrexial Reactions during Haemodialysis
}

\author{
P. J. A. ROBINSON, S. M. ROSEN
}

\section{Clinical Features}

Most reactions follow a typical pattern. The onset, with shivering and a rise in temperature, occurs within one to two hours of starting dialysis. Vomiting, back pain, and hypotension are the most frequent symptoms. These may persist for one to two days, but usually subside by the end of the dialysis period. In this series severe reactions resulted in hospital admission on 25 occasions. Prolonged hypotension was associated with clotting in the arteriovenous shunt on 10 occasions.

In most instances treatment was restricted to symptomatic measures. These included oral paracetamol or intravenous dihydrocodeine for headache and back pain, diazepam for severe rigor and agitation, papaverine or thymoxamine for shunt vein spasm, cooling by means of fans or tepid sponging, and replacement of fluid losses due to vomiting or diarrhoea. Intravenous hydrocortisone $100-200 \mathrm{mg}$ given at the start of dialysis did not prevent reactions. Patients who developed pyrexial reactions in association with general or local infection were treated with appropriate antibiotics, as were those who produced positive blood cultures.

\section{Incidence}

Four hundred and fifty reactions occurred in 468 patientmonths, giving an overall incidence of 0.96 reaction per patient-month. Though every patient who has had more than 10 dialyses has had one or more pyrexial reactions, the incidence of reactions varies widely in the patient population. In those who have had over 100 dialyses the incidence varies between $2 \%$ and $23 \%$, with a mean of about $10 \%$. The severity and duration of symptoms associated with reactions show a wide variation not only between different patients but between different occasions in the same patient.

The incidence of reactions does not correlate with age or sex, but patients have more frequent reactions in the first three months than subsequently. Patients undergoing dialysis at home who use similar techniques and equipment to those used in hospital also experience pyrexial reactions. They do not record their temperature during dialysis unless they are symptomatic, and this may account for a small decrease in the recorded incidence of reactions when patients transfer from hospital to home dialysis. There is, however, a pronounced difference in the incidence between home and hospital dialysis (Table I), which suggests a variable environmental influence.

Reactions occur in epidemics lasting from one day to several weeks. Analysis of the day-to-day frequency of reactions fails to show a random distribution.

\section{Investigations}

Blood Cultures.-The possibility that these reactions are related to the introduction of bacteria from the dialyser into the patients' blood was investigated by collecting blood culture specimens from patients during each of 450 pyrexial reactions. Cultures were also made of specimens taken at the same time from patients on dialysis who were not having reactions. The controls were representative of all groups. When a positive culture was obtained from a patient further speci-
Department of Renal Medicine, Leeds University (St. James's) Hospital Leeds LS9 7TF

P. J. A. ROBINSON, M.B., M.R.C.P., Clinical Scientist

S. M. ROSEN, M.B., M.R.C.P. (LOND., ED.), Physician in Charge 
TABLE I-Incidence of Pyrexial Reactions in Home and Hospital Patients

\begin{tabular}{|c|c|c|c|c|}
\hline & & & $\begin{array}{c}\text { Home } \\
\text { Patients (12) }\end{array}$ & $\begin{array}{c}\text { Hospital } \\
\text { Patients (39) }\end{array}$ \\
\hline $\begin{array}{l}\text { Total number of reactions } \\
\text { Total number of patient-months } \\
\text { Incidence (reactions/patient-month) }\end{array}$ & $\begin{array}{ll}\cdots & \cdots \\
\cdots & \cdots \\
\cdots & \cdots\end{array}$ & $\begin{array}{l}\cdots \\
\cdots\end{array}$ & $\begin{array}{l}27 \\
66 \\
0 \cdot 4\end{array}$ & $\begin{array}{l}423 \\
402 \\
1 \cdot 1\end{array}$ \\
\hline
\end{tabular}

TABLE II-Organisms Grown from Blood Cultures

\begin{tabular}{|c|c|c|c|c|c|c|c|c|}
\hline & & & & & & Rigors & Controls & Repeats \\
\hline $\begin{array}{l}\text { Streptococcus } \\
\text { Diphtheroids.. } \\
\text { Staphylococcus } \\
\text { Bacillus cereus } \\
\text { Paracolon } . \\
\text { Staph. aureus } \\
\text { B-haemolytic s } \\
\text { Escherichia col } \\
\text { Bacillus subtilis } \\
\text { Alcaligenes faee } \\
\text { Pseudomonas p. } \\
\text { Str. viridans } \\
\text { Micrococcus } \\
\text { Chromobacter } \\
\end{array}$ & $\begin{array}{l}\text { lis } \\
\ldots \\
\text { is } \\
\ldots \\
\ldots \\
\ldots \\
\text { tococcus } \\
\ldots \\
\ldots \\
\text { anea } \\
\ldots \\
\cdots \\
\cdots\end{array}$ & $\begin{array}{l}\ldots \\
\cdots \\
\cdots \\
\cdots \\
\text { s } \\
\cdots \\
\cdots \\
\cdots \\
\cdots \\
\cdots \\
\end{array}$ & $\begin{array}{l}\ldots \\
\cdots \\
\cdots \\
\cdots \\
\cdots \\
\cdots \\
\cdots \\
\cdots \\
\cdots \\
\cdots \\
\cdots\end{array}$ & $\begin{array}{l}\cdots \\
\cdots \\
\cdots \\
\cdots \\
\cdots \\
\cdots \\
\cdots \\
\cdots \\
\cdots \\
\cdots \\
\cdots\end{array}$ & $\begin{array}{l}\ldots \\
\cdots \\
\cdots \\
\cdots \\
\cdots \\
\cdots \\
\cdots \\
\cdots \\
\cdots \\
\cdots \\
\cdots\end{array}$ & $\begin{array}{r}11 \\
7 \\
3 \\
6 \\
1 \\
1 \\
3 \\
2 \\
- \\
1 \\
1 \\
1 \\
1\end{array}$ & $\begin{array}{l}\overline{2} \\
4 \\
\overline{1} \\
\overline{1} \\
\overline{2} \\
1 \\
\overline{1} \\
=\end{array}$ & $\begin{array}{r}15 \\
3 \\
4 \\
8 \\
2 \\
2 \\
\frac{3}{2} \\
= \\
=\end{array}$ \\
\hline Chromobacter & & & & & & 38 & 10 & 37 \\
\hline
\end{tabular}

mens for culture were taken at the same time of each subsequent dialysis until several negative results were obtained. After a positive culture result, 137 repeat cultures were performed in nine patients, and 29 of those from three patients produced positive results. Two patients had persistent positive blood cultures-one had signs of subacute bacterial endocarditis and in the other the source of infection was not found. In neither patient was there an increased incidence of pyrexial reactions. The different organisms isolated from blood culture specimens are shown in Table II. The results of the blood cultures showed that: (a) positive cultures are found as often in controls as in patients undergoing pyrexial reactions, $(b)$ the frequency of positive cultures is not related to the frequency of reactions, $(c)$ the incidence of fever and symptoms during reactions is the same in patients producing positive and negative cultures, and $(d)$ a wide variety of organisms had been isolated from the blood of both groups of patients. Repeatedly positive cultures in two patients were not associated with repeated pyrexial reactions.

Type of Monitor.-Three types of dialysate fluid preparation and monitoring equipment have been used. Firstly, a central distribution system with Watson-Marlow monitors at each bed; secondly, individual patient machines of the Lucas type; and thirdly, the Dylade $B$ type of individual patient machine. The first two systems were sterilized with formalin, while the Dylade B machines were heat-sterilized. The incidence of reactions was the same for each of the three different types of equipment.

Use of the R.S.P. Coil Dialyser.-Reactions also occur with the R.S.P. dialyser when using a pyrogen-free* disposable coil which is sterilized with ethylene oxide. We do not normallv use the coil dialyser for maintenance dialysis, but we selected three patients who were having frequent reactions for a trial on coil and Kiil systems for a month. Reactions occurred in 3 out of 20 dialyses with the Kiil and 4 out of 20 with the coil. Of the four reactions seen during dialysis with the R.S.P. coil system, three presented an unusual feature in that they developed during the final hour of an eight-hour treatment. The symptoms, however, were similar to those previously recorded. Blood cultures on each occasion were sterile.

Deionization of Mains Water.-An Elgastadt deionizer producing water with a conductivity of $1 \mu \mathrm{mho} / \mathrm{cm}^{2}$ was fitted to the water supply of one of the six bed stations in the dialysis unit. The incidence of reactions in the four patients dialysed in this station was not different from the incidence in other hospital patients during the same period.

*Pyrogen test. British Pharmacopoeia
Albuminoid Nitrogen Content of Tap-Water.-The albuminoid nitrogen content of water samples was measured by the Leeds Corporation Water Works Department, using the approved method (Fournal of the Institute of Water Engineers, October 1949). The levels in samples taken from the dialysate supply taps (mean 0.044 p.p.m., range $0.036-0.056$ ) and in samples from the drinking-water tap (mean 0.046 p.p.m., range $0.036-0.058$ ) did not differ significantly from the levels in samples taken from the water leaving the filtration plant (mean 0.045 p.p.m., range 0.035-0.056). There was no correlation between the monthly incidence of pyrexial reactions and the levels of albuminoid nitrogen in the mains water supply.

\section{Discussion}

Pyrexial reactions during haemodialysis have been widely recognized, but there is little published information on their nature and cause. After excluding those associated with demonstrable infection, blood transfusion, or drug sensitivity, there remains a large group of unexplained reactions with a common pattern. These do not occur between dialyses and are not associated with peritoneal dialysis, with manipulation of the arteriovenous shunt-for example, in declotting-or with the injection of heparin. Blood pumps are not normally used during dialysis, and there is no evidence for haemolysis as a contributing factor.

The clinical pattern of the reactions, with rigor and fever following a short latent period after starting dialysis, is similar to that produced in experimental subjects by the intravenous injection of endotoxins isolated from Gram-negative bacteria (Bennett and Cluff, 1957). Though such endotoxins are antigenic, the presence of specific antibody in subjects repeatedly exposed does not modify the symptomatic response to the toxin. Gazenfield-Gazit and Eliahou (1969) found antibodies to dialysate bacterial endotoxins in a group of dialysis patients. Dialysis patients show a consistent susceptibility to reactions, even after many previous exposures. This contrasts with the decrease in severity of reactions seen with repeated injections of live bacteria. Further evidence against the role of live bacteria in producing dialysis reactions is shown by the lack of correlation with positive blood cultures.

Jones et al. (1970) suggested that dialysis reactions are produced by the entry into the blood of bacteria from the dialysate compartment and demonstrated the persistence of bacteria in Kiil boards exposed to $2 \%$ formaldehyde. The incidence of reactions in our patients did not correlate with the presence of bacteria in samples of priming saline taken from the blood compartment of the dialysers. We found a random distribution of reactions among individual dialysers. The frequency of reactions was the same whether the Kiil boards were sterilized with $3 \%$ acetic acid or $2 \%$ formaldehyde, and whether the monitoring equipment was sterilized with formaldehyde or heat.

There is a definite epidemic pattern to the incidence of reactions, suggesting that the cause is a variable environmental factor affecting all patients and unrelated to differences in dialysis techniques. The factor which most readily fits these criteria is tap-water, which is known to be a source of pyrogens. Tan-water is used for soaking the membranes during the assembly of the Kiil dialysers. There are two possible rnutes of entry into the blood for the pyrogenic substance. Either it is present on the blood compartment surface of the membranes at the start of dialysis or it must cross the membrane from the dialysate fluid. If the tap-water in which the membranes are soaked is heavily contaminated bacterial debris may be introduced into the circulation at the start of dialysis.

The use of the disposable coil kidney does not eliminate reactions, and here it seems likely that the pyrogen crosses 
the membrane during dialysis. The later onset, during dialysis, of reactions in coil-dialysed patients may be related to an increase in the bacterial population of the dialysate fluid and/or changes in the permeability of the membrane during dialysis.

Improved general health in home dialysis patients, together with less thorough data recording, may contribute to their lower incidence of reactions. The use of ion-exchange resins does not necessarily remove pyrogens from the water supply, and the resin itself may act as a reservoir of bacterial products. In our small group the use of a deionizer did not abolish pyrexial reactions. Biagini et al. (1970) reduced the incidence of reactions by pretreating the dialysate fluid with activated charcoal.

Prevention of reactions is otherwise limited to ensuring adequate sterilization of equipment and decontamination of the tap-water tanks used for soaking the dialysis membranes for the Kiil kidney. Treatment of the reactions is mainly symptomatic.

We wish to thank Mr. Neale and Mrs. Dunn, of the bacteriology department, St. James's Hospital, who examined the culture specimens; Mr. J. R. Roberts, of the Leeds City Water Works Department, for the tap-water analyses; and Sister P. A. Farrar and the nursing and technical staff of the regular dialysis unit for their untiring support and care of the patients.

\section{References}

Bennett, I. L., and Cluff, L. E. (1957) Pharmacological Reviews, 9, 427. Biagini, M., Rindi, P., Rizzo, G., and Giovanneti, S. (1970). Proceedings of the European Dialysis and Transplant Association, 7, 467.

Gazenfield-Gazit, E., and Eliahou, H. E. (1969). Israel fournal of Medical Sciences, 5, 1032.

Jones, D. M., Tobin, B. M., Harlow, G. R., and Ralston, A. J. (1970), British Medical fournal, 3, 135

\title{
Abnormal Chemoreceptor Response to Hypoxia in Patients with Tabes Dorsalis
}

\author{
R. J. COURTENAY EVANS, M. K. BENSON, D. T. D. HUGHES
}

British Medical fournal, 1971, 1, 530-531

\section{Summary}

Nine patients with tabes dorsalis and one patient with diabetic autonomic neuropathy were subjected to hypoxia to test the integrity of their carotid chemoreceptors. Ventilation and pulse rate changes were monitored and compared with those of a group of normal subjects of comparable age and sex. Four patients had a completely negative response to hypoxia, and the changes in ventilation in the group of patients as a whole were significantly less than in the control subjects. The results indicate that some patients with autonomic denervation are unable to adjust their respiratory system in response to hypoxia.

\section{Introduction}

The carotid and aortic chemoreceptors respond to hypoxia in normal subjects by causing a reflex increase in pulse rate and ventilatory volume (Dripps and Comroe, 1947). These organs are sensitive primarily to lowering of arterial oxygen tension but do have less sensitivity to other stimuli. The reflex arc involved is innervated by visceral afferent nerves from the chemoreceptors to the brain stem, and then by sympathetic nerves to the heart and by somatic efferent nerves to the respiratory muscles.

The carotid and aortic baroreceptors are sensory receptors

London Hospital, London E1 1BB

R. J. COURTENAY EVANS, M.B., M.R.C.P. (Present appointment : Senior Medical Registrar, Addenbrooke's Hospital, Cambridge)

M. K. BENSON, M.B., M.R.C.P., Wellcome Research Fellow

D. T. D. HUGHES, B.SC., M.R.C.P., Senior Lecturer in Medicine for reflexes controlling blood pressure. Sharpey-Schafer (1956) showed that in fully developed tabes dorsalis the baroreceptor reflexes may be absent owing to the disease process affecting the afferent nerves involved in the reflex arc. In a later study (Sharpey-Schafer and Taylor, 1960) it was shown that in 31 out of 337 diabetics there were either defective or absent baroreceptcr reflexes. These abnormalities were associated with generalized autonomic denervation. Though there are several reports on the effects of autonomic denervation on the baroreceptor reflex, there is no report of the chemoreceptor reflexes in this condition. The results of surgical denervation of the carotid chemoreceptors, however, have been reported in two patients with asthma. After denervation they failed to respond to hypoxia (Holton and Wood, 1965). It was therefore decided to assess the effect of hypoxia on patients with tabes dorsalis and diabetic autonomic neuropathy.

\section{Methods}

Dripps and Comroe (1947), showed that the percentage of oxygen in inspired air has to be dropped to $10 \%$ before there is a constant pronounced increase in minute volume and pulse rate. The hypoxic stimulus used in the present study was therefore $10 \%$ oxygen and $90 \%$ nitrogen and ventilation was measured by the "bag in box" method (Donald and Christie, 1949). The alveolar carbon dioxide tension was not controlled in the present preliminary studies. Pulse rate was monitored by means of an electrocardiogram. Subjects were connected to the bag-box system and electrocardiograph. They became accustomed to breathing room air through the mouthpiece and valve until the tidal volume became constant. They were then turned on to the bag mixtures: room air $(20.9 \%$ oxygen) for three minutes and $10 \%$ oxygen for three minutes. The respiratory rate, tidal volume, minute volume, and pulse rate were all recorded. In normal subiects there was a rapid change in ventilation on switching to the low oxygen mixture. The ventilation in the second and third minutes was similar, and that during the third minute was measured and compared with the third minute breathing air. 